\title{
Depredación de ganado por jaguar y otros carnívoros en el noreste de México
}

Juan L. Peña-Mondragón ${ }^{1 *}$ y Alicia Castillo ${ }^{1}$

\section{Abstract}

The principal factors that contribute to the conservation of large carnivorous mammals are, the increase in human density, the amount of remnant natural habitat, land use change and hunting (of the species and their natural preys). In order to take effective conservation actions is necessary to understand all the dimensions of the human-carnivore conflict. One alternative is to assess the economic damage caused by carnivores on cattle a herd which is a major cause of their persecution and elimination. Damage assessed by jaguar in southern of Nuevo Leon. At the same time, we evaluated the economic damage of black bear, coyote, cougar, bobcat and gray fox in order to compare the economic value of damages to livestock. Eighty people were surveyed in 60 rural communities. Economic damages in livestock (in USD) were 134,253 in 1992-2010. By species these were: black bear 43,077; jaguar 39,016; cougar 17,057; coyote 28,492; bob cat 4,095 and gray fox 2,514. Knowing the damage that the jaguar and other carnivores have on the economy of peasant's families is essential for the design of mitigation strategies that lead to conflict and that conduce to achieving the conservation of the jaguar.

Keywords: Panthera onca, human - wildlife conflict, conservation, Nuevo León.

\section{Resumen}

El aumento de la densidad humana, la cantidad de hábitat natural remanente, el uso de suelo y la incidencia de cacería, ya sea de subsistencia o furtiva (de la especie y de sus presas naturales), son factores que contribuyen a la conservación o eliminación de los grandes mamíferos carnívoros. Para poder emprender acciones de conservación efectivas, es necesario entender el problema en todas sus dimensiones. Una de ellas es poder contabilizar los daños económicos que causan los carnívoros en los hatos ganaderos que es una de las principales causas de su persecución y eliminación. En el presente trabajo evaluamos los daños causados por jaguar en el sur de Nuevo León. Al mismo tiempo, se evaluaron los daños económicos por oso negro, coyote, puma, gato montés y zorra gris para comparar el valor económico de los daños sobre la ganadería. Se encuestaron 80 personas en 60 comunidades rurales. Los daños económicos (en dólares Americanos) en la ganadería ascienden a 134,253 en el lapso de tiempo 19922010. Por especie corresponden a: oso negro 43,077; jaguar 39,016; puma 17,057; coyote 28,492; gato montés 4,095 y zorra gris 2,514. Conocer los daños que el jaguar

${ }^{1}$ Laboratorio de Comunicación para el Manejo de Ecosistemas. Centro de Investigaciones en Ecosistemas (CIEco), UNAM-Campus Morelia. Antigua Carretera a Pátzcuaro 8701. Morelia, Michoacán 58190, Mexico. E-mail: jlpena@ cieco.unam.mx (JLP-M), castillo@cieco.unam.mx (AC)

*Corresponding autor 
y otros carnívoros generan en la economía de las familias campesinas es esencial para el diseño de estrategias de mitigación del conflicto y que lleven a lograr la conservación de las especies.

Palabras clave: Panthera onca, carnívoros, conflicto humano-vida silvestre, conservación, Nuevo León.

Introducción

El aumento de la población humana a nivel mundial y la demanda cada vez mayor de recursos naturales, ha tenido como consecuencia la reducción de las áreas de distribución de la mayoría de las especies, aislando a la biodiversidad a espacios pequeños para su conservación y forzando a la vida silvestre a vivir cerca de las poblaciones humanas (Hoogesteijn 2003; Inskip y Zimmerman 2009). Lo anterior tiene como consecuencia que se acentúe el traslape entre los espacios utilizados por los seres humanos y el hábitat de algunas especies silvestres y por ende el desarrollo de conflictos, entre ellos la competencia por los recursos. Los grandes mamíferos carnívoros son especies que resienten más esta competencia ya que son sensibles a los cambios en el ecosistema, uno de los principales hoy en día es la deforestación (Hoogesteijn 2003). Esta genera un aumento de la frontera agrícola o la disminución de hábitat que es uno de los factores que desencadena la depredación de ganado (bovino: Bos sp.; caprino: Capra sp.; ovino: Ovis orientalis aries; porcino: Sus crofa domestica; equinos: Equus sp.), uno de los principales conflictos entre el hombre y los grandes mamíferos carnívoros (Hoogesteijn 2003).

Teniendo como resultado la persecución y eliminación de los grandes carnívoros por los dueños de ganado (Hoogesteijn 2003; Garrote 2012). Esto incluye la extirpación de estas especies en los sitios ubicados fuera de las áreas protegidas (Nowell y Jackson 1996).

Cabe señalar, que la gran mayoría de los casos de depredación de ganado por carnívoros silvestres, refleja algún tipo de desequilibrio (falta de espacio, falta o disminución de presas, aumento de las poblaciones humanas), en el ecosistema local (Hoogesteijn 2003). En México, entre las especies que depredan ganado se puede mencionar al coyote (Canis latrans), oso negro (Ursus americanus), puma (Puma concolor), jaguar (Panthera onca); así como felinos pequeños [gato montés (Lynx rufus), ocelote (Leopardus pardalis), margay o tigrillo (Leopardus wiedii) y jaguarundi (Puma yagouaroundi; Servín y Huxley 1991; Aranda et al. 2002; Peña-Mondragón 2011; Amador et al. 2013)]. Entre estas especies, el jaguar es una de las especies más afectadas; ha desaparecido en más del 60\% de su distribución original en México (Hoogesteijn y Hoogesteijn 2011, Ceballos et al. 2006).

El ganado no es considerado una presa natural (nativa de su distribución) del jaguar, pero a pesar de ello aparece como un componente de su dieta en América Latina teniendo repercusión en la economía de las familias rurales. En Venezuela se reportan pérdidas cercanas a los USD 36,000 (Scognamillo et al. 2002). En el Cerrado, Brasil las pérdidas por jaguar representan el $80 \%$ de las pérdidas en las fincas (Crawshaw y Quigley 2002). En Pantanal, Brasil las pérdidas se acercan a los USD 28,500 tan sólo en novillos y becerros (De Azevedo 2008; Oliveira 1994). Al sur de Brasil se reportan pérdidas por USD 699,670 (Mazzolli et al. 2002). En el centro oeste de Brasil los reportes ascienden a USD 1.4 millones (Palmeira et al. 2008). En Centro América las pérdidas 
en Costa Rica son cercanas a los USD 60,000 (Moreno y Olmos 2008; Saenz et al. 2002). En Colombia las pérdidas son reportadas de manera general como bajas (Garrote 2012). Para Guatemala las pérdidas reportadas ascienden a USD 14,736 (Soto-Shoender y Giuliano 2011). Todo lo anterior, tiene un común denominador: la persecución y eliminación del jaguar por los dueños del mismo (Garrote 2012). En México hay pocos datos publicados que aborden el impacto económico del jaguar u otros carnívoros en la ganadería, se cuenta con información en Chiapas, Querétaro, Sonora y Yucatán (Ortega 2006; Rosas-Rosas et al. 2008; Hernández 2009 y, Amador-Alcalá et al. 2013). Para poder diseñar manuales de manejo ganadero, o poder emitir recomendaciones para prevenir los ataques en el ganado, es necesario obtener información precisa de cada depredador, tanto en preferencias de ataque como en la correcta identificación del mismo (Rosas-Rosas et al. 2008). Uno de los aspectos importantes al caracterizar los daños del ganado y la correcta identificación de los depredadores y dimensionarlo con el impacto familiar, es su potencial implicación en la mejora del "seguro de muerte por el ataque de depredadores" (Confederación Nacional Ganadera-SAGARPA). Actualmente el seguro ganadero sólo cubre ganado bovino reproductivo y de doble función (para producir carne y además otros derivados como leche, queso, cuero), bovino lechero, ovino y caprino.

Los estudios hasta el día de hoy muestran que la depredación de equinos y porcino es también un detonante que puede encrudecer el conflicto.

El presente trabajo buscó documentar el impacto económico del jaguar en la ganadería en dos municipios de Nuevo León, recabando datos sobre esta especie paraguas, clave, bandera y emblemática (jaguar; SEMARNAT 2009; Conde et al. 2011) y otros carnívoros (oso negro, puma, coyote, gato montés y zorra gris), ya que en ocasiones el jaguar es culpado por los daños causados por otros carnívoros y en consecuencia eliminado. En esta zona, el jaguar ha sido poco estudiado, sólo existe un trabajo publicado y aborda la distribución y estado de conservación del jaguar (Rosas-Rosas y López-Soto 2002), excluyendo la relación de la especie y las poblaciones humanas. El fin último del trabajo es aportar elementos que permitan contribuir a la formulación e implementación de estrategias de mitigación y prevención de la depredación de ganado por jaguar por las propias comunidades. Así como aportar datos que contribuyan a mejorar los programas y políticas dedicados a mitigar el conflicto existente. Ambas con el objetivo de conservar a largo plazo los carnívoros, incluido el jaguar.

\section{Material}

y Métodos

Área de estudio. Comprende dos municipios del sur de Nuevo León: Aramberri y General Zaragoza (Fig. 1), pertenecen a la Subprovincia Fisiográfica Gran Sierra Plegada y se encuentran inmersos en el macizo montañoso de la Sierra Madre Oriental, entre los límites de Nuevo León y Tamaulipas. La Sierra Madre Oriental se caracteriza por presentar terrenos muy accidentados, en forma de sierras paralelas en dirección nornoroeste a sursureste, que alcanzan en promedio los 2,000 msnm, y en donde se ubican los picos más altos del estado de Nuevo León, como el Cerro del Potosí, La Ascensión y Peña Nevada con más 3,500 msnm. Los cauces que se forman entre las sierras, llevan arroyos de diversa importancia cuyo desagüe natural se dirige hacia el este (Alanís et al. 1996). El relieve, el contraste latitudinal debido a la topografía de la Sierra Madre 
Oriental, la exposición a la energía solar, los diferentes tipos de suelo y su capacidad de retener humedad, así como los regímenes de precipitación que son influenciados por la penetración de masas de aire húmedo provenientes del Golfo de México, regulan los diferentes tipos de vegetación de esta zona, compuesta principalmente por bosques de pinos, encinos, bosques mixtos y otros tipos de coníferas (INEGI 1986; Alanís et al. 1996).

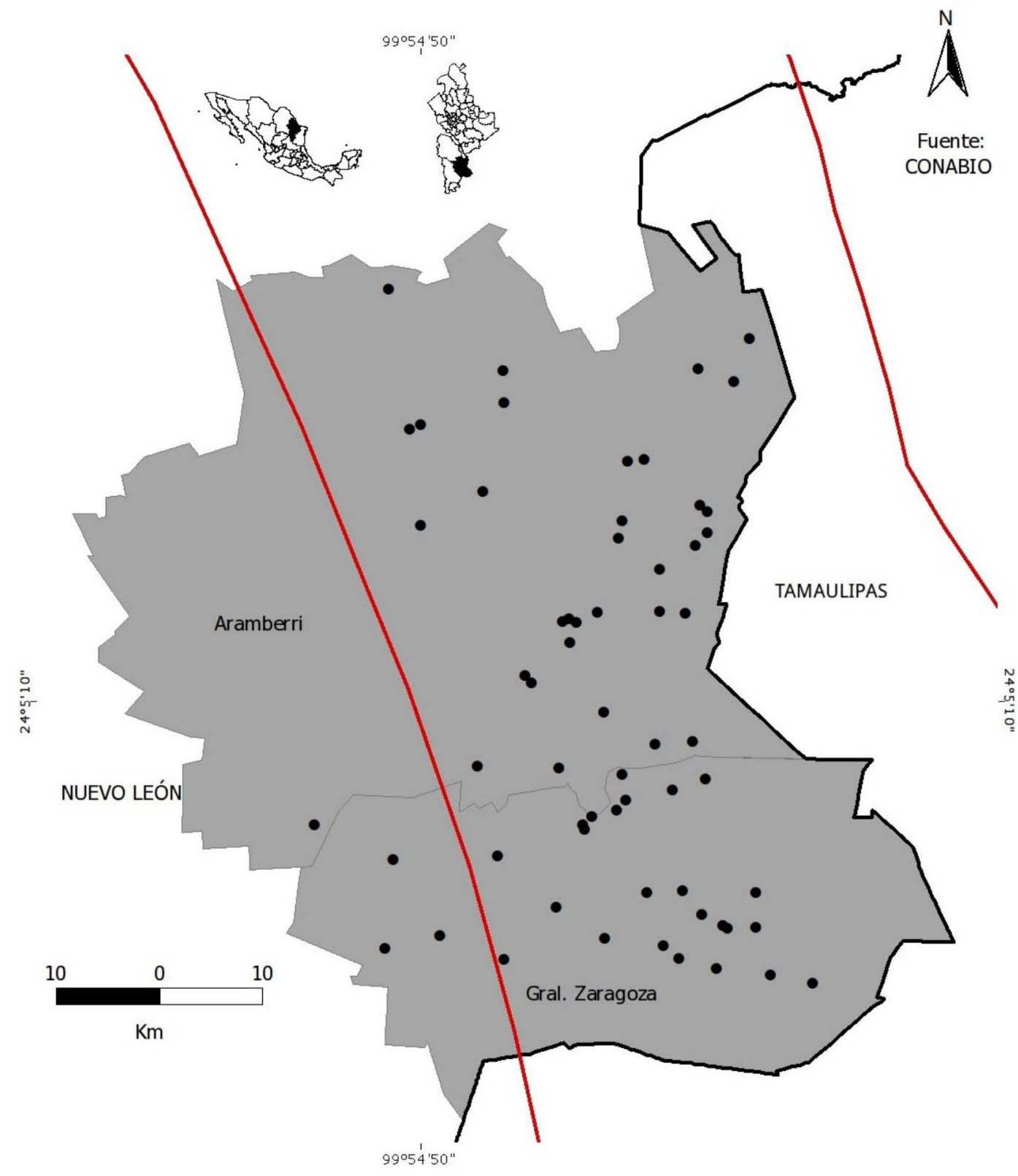

Figura 1. Localización geográfica del área de estudio, se muestran los municipios Aramberri y General Zaragoza en el sur de Nuevo León (gris oscuro). (•) comunidades visitadas durante el desarrollo del trabajo. ( $(\eta)$ Subprovincia Fisiográfica Gran Sierra Plegada, ( $)$ división municipal y estatal ( ().

Enfoque de investigación. El enfoque de investigación es de corte socio-ecológico, debido a que interesa entender el conflicto existente entre los grupos humanos, sus actividades productivas y las afectaciones de los animales silvestres (Drury et al. 2010).

Este enfoque adquiere cada vez más auge en las investigaciones ambientales (Berkes y Folke 2000), ya que se basa en la relevancia de aceptar que en la actualidad las sociedades humanas no solamente dependen de los bienes y servicios que obtienen de los ecosistemas (MEA 2003), sino también reconoce que los ambientes naturales 
en el planeta, están impactados y continuamente influenciados por las intervenciones humanas (Vitousek et al. 1997).

Instrumentos de investigación. Se utilizaron dos herramientas principales para el levantamiento de datos: la encuesta (compuesta de preguntas abiertas y cerradas; LópezRomo 1998; Hernández-Sampieri 1991) y la observación participante (Tarrés 2004). El muestreo se hizo usando el método conocido como "bola de nieve" (Sánchez-Serrano 2004). Consiste básicamente en que al terminar de realizar una encuesta se le pregunta a la persona, por otra persona que pueda aportar información referente al tema de la investigación y que sea posible encuestarla también (Sánchez-Serrano 2004). En este caso interesó obtener información de personas que tuvieran problemas similares de pérdida de ganado y así sucesivamente. Esto llevó a trabajar, por ejemplo, en localidades que no se tenían contempladas al inicio de la investigación.

Selección de los sitios de trabajo. Las comunidades iníciales a incluir en el trabajo, se seleccionaron de acuerdo a los 26 registros que existen publicados de jaguar en la zona (Rosas-Rosas y López-Soto 2002) y con base en la experiencia del primer autor en el área de estudio. Tomando como base estos registros, se trazó una radio de $25 \mathrm{~km}^{2}$ (tomando como referencia el ámbito hogareño de un jaguar hembra reportado para Chamela, Jalisco; Núñez et al. 2002) a partir de cada registro y se seleccionaron las localidades (rurales) que estuvieran dentro de este radio, seleccionando inicialmente las más cercanas a los registros para el inicio del muestro. Paralelamente, en las reuniones anuales de las Asociaciones Ganaderas Municipales, se presentó el trabajo de investigación (una reunión por municipio); se solicitó autorización y apoyo voluntario para levantar un padrón de ganaderos con problemas de pérdidas de ganado por jaguar y otros carnívoros. Con base en las dos estrategias se obtuvo un padrón de personas dispuestas a colaborar con el proyecto, mismas que se visitaron a lo largo del trabajo.

El criterio de elegibilidad de los actores sociales fue que hubieran sufrido daños por el jaguar y/o otras especies de carnívoros y que recordaran las fechas y cantidades de animales perdidos aproximadamente. Dentro del contexto de trabajo un actor social se define como: aquellas personas que tienen la capacidad de procesar la experiencia social e idear maneras de hacer frente a distintas situaciones que les presente la vida, incluso dentro de escenarios conflictivos como lo es la decisión de eliminar al jaguar de su propiedad privada o comunal (Long 1992).

Inicialmente el trabajo estuvo dirigido a jaguar, y en etapa temprana del trabajo de campo se decidió ampliar el rango de especies de carnívoros incluidas en las encuestas. Lo anterior por la complejidad que implica trabajar sólo con jaguar en una zona en donde se niega su presencia. En donde también es perseguido por los pobladores como medida preventiva y/o correctiva del conflicto sabiendo que existe prohibición de su cacería (NOM-059-SEMARNAT-2010). Entonces el preguntar por diferentes especies de carnívoros facilitó el flujo de información referente al jaguar, debido a qué los encuestados no sentían presión por responder sólo cuestiones relacionadas con el jaguar.

Aplicación de encuestas. El formato de encuesta fue modificado del trabajo de Bueno (2004) de depredación de ganado por pumas en Cañón de Santa Elena, Chihuahua, 
México. La encuesta estuvo dirigida a actores sociales clave como jefes de familia y/o personas involucradas en el manejo de tierras. Cada cuestionario consistió en preguntas cerradas para obtener datos puntuales sobre los daños en el ganado debidos a los carnívoros presentes en el área (e. g. jaguar, puma, gato montés, oso negro, coyote y zorra gris). Se incluyeron también, preguntas abiertas que permitieron indagar en los patrones de depredación para la correcta identificación del depredador responsabilizado del ataque (Wade y Browns 1982; Hoogestein y Hoggestin 2011). El período contemplado para registrar los eventos de depredación fue de 2007 al 2010. En el caso de que alguna persona recordara eventos anteriores a este periodo, se agruparon en los siguientes períodos: 1992 - 2001 y 2002 - 2007. Debido a que al pasar el tiempo la información puede carecer de veracidad (Amador et al. 2013), sólo se contemplaron los eventos de depredación anteriores a 2007 aquellos que se consideran traumáticos para las personas. Esto por la intensidad del evento, por alguna fecha de importancia para el encuestado o por la eliminación del jaguar responsable que es considerado un trofeo. La encuesta se aplicó a manera de charla y dentro de un ambiente que no resultara incómodo para la persona (e. g. su área de trabajo, su casa o recorriendo los sitios donde sucedieron los ataques al ganado por los depredadores).

La conducción de la entrevista consistió en iniciar con dos preguntas guías ¿usted ha sufrido daños en su ganado por carnívoros en los últimos años? y ¿Por cuál de ellos ha sufrido pérdidas? Explicando previamente el objetivo del trabajo y haber presentado el equipo de trabajo y mostrado los permisos comunitarios correspondientes. A partir de entonces se indago en los carnívoros responsables y en asegurar la veracidad de los datos en concordancia con patrones de depredación típicos de los carnívoros mencionados.

Validación de resultados. Al finalizar el proyecto se organizaron dos reuniones en comunidades claves con el objetivo de devolver la información y obtener su visión acerca de las dimensiones de los daños que se obtuvieron en el trabajo. Así mismo, el quipo de trabajo se presentó de nuevo en las reuniones anuales de las Asociaciones Ganaderas Locales de ambos municipios trabajados y se informó al público de los resultados del trabajo y se entregó un folleto a cada uno de los asistentes con los resultados principales.

Consideraciones éticas. Aunque se recomienda en la literatura de investigación social, registrar lo más fiel posible lo que una persona dice cuando se hace una entrevista (hablar con las personas es una de las formas más utilizadas para obtener información), se decidió no utilizar ningún instrumento de registro de audio. Esto debido a que se tuvieron experiencias de desconfianza previas en dos visitas piloto al sitio de trabajo.

El procedimiento general que se siguió fue que se explicó con detalle el propósito de la investigación y se les aseguró a los participantes que la información que las personas brindaran, no se utilizaría para ningún tipo de denuncia, que se mantendría el anonimato de ellas y que el uso de la información sería exclusivamente con fines de investigación.

Se resaltó la confidencialidad de las entrevistas y que los resultados serían dados a conocer posteriormente a los participantes. Se firmó una carta compromiso de entrega de resultados con cada una de las autoridades ejidales. En el inicio de cada encuesta y en el desarrollo de la misma se les mencionó a cada uno de los entrevistados, que el equipo de trabajo no era portador de apoyos económicos, que no se repondrían animales 
depredados por carnívoros y que no se pertenecía a alguna agencia gubernamental.

Análisis. Para las preguntas ¿usted ha sufrido daños en su ganado por carnívoros en los últimos años? y ¿por cuál de ellos ha sufrido pérdidas?; los resultados se expresan en porcentajes e intervalos de confianza del 95\% (JMP 7.0 SAS Institute 2007). Los porcentajes o proporciones pueden no sumar $100 \%$ debido a que hay preguntas que pueden ser no excluyentes o los encuestados podían proporcionar varias respuestas a una sola pregunta.

Para la valoración económica de los daños, se asignó un valor monetario para cada individuo de los diferentes tipos de ganado. El valor unitario se obtuvo de las encuestas, al término de cada una se preguntó cuál era el valor económico de los animales perdidos y se calculó una media para obtener los valores unitarios (Tamang y Baral 2008). Se consideró el valor proporcionado por las encuestas, porque para los pobladores este significa el valor real de su pérdida y es proporcional al nivel de impacto en su economía familiar. A su vez en el área de trabajo el precio de compra del mercado es diferente al pagado que llega a los propietarios, sobre todo por la presencia de intermediarios que pueden manejar los precios y en ocasiones pagar un valor menor al establecido. Los tipos de ganado analizados y su valor unitario fueron (en dolareas americanos): aviar (8.33), bovino (201.19), caballos (81.78), mulas (297.40), burros (31.60) caprino (39.11), ovino (40.30) y porcino (148.70), el tipo de cambio utilizado fue 13.45, de acuerdo al Banco de México el día 19 junio de 2013. Durante el trabajo los encuestados no fueron cuestionados por pérdidas relacionadas a enfermedades, parásitos, factores climáticos o robo. El objetivo primordial fue evaluar exclusivamente las pérdidas por depredación.

\section{Resultados}

Se visitaron un total de 60 localidades en los municipios de Aramberri y General Zaragoza, Nuevo León. Se encuestó a 80 personas con edades que fluctuaron en 24 y 82 años. El periodo de tiempo en el que se registraron los ataques fue de 1992 a 2010. De los 80 encuestados, el 90.23\% (IC: 80.45 - 95.48\%) afirmó haber tenido pérdidas por carnívoros presentes en la región de estudio (e. g. oso negro, zorra gris, coyote, gato montés, puma o león y jaguar o tigre). Respecto a los daños económicos generados por cada uno de las especies de carnívoros, el 43.54\% (IC: $37.94-55.90 \%$ ) de los encuestados afirmó haber tenido pérdidas por oso negro; un 32.25\% (IC: $21.95 \%$ - 44.63\%) de pérdidas por puma y el 29.03\% (IC: 19.22 - 41.28\%) responsabilizó al jaguar, esto respecto a los grandes carnívoros (peso superior a $5 \mathrm{~kg}$, Cortés 2009). Respecto a los carnívoros medianos (peso entre 150 y 5 kg; Cortés 2009), el 58.06\% (IC: $45.66-69.52 \%$ ) de los encuestados afirmó haber sufrido pérdidas por coyote; un 19.35 (IC: 11.43 - 30.85\%) responsabilizó al gato montés y el 30.64\% (IC: 20.58 - 42.96\%) reconoció a la zorra gris como el depredador responsable.

Los daños en la ganadería por especie se agrupan de la siguiente manera (en dólares americanos): el oso negro fue la especie con mayor monto registrando $(43,077)$, el segundo mencionado fue el jaguar $(39,016)$ y en tercer lugar coyote $(28,492)$. Los últimos carnívoros mencionados fueron el puma $(17,057)$, el gato montés $(4,095)$ y la zorra gris $(2,514)$. Todo lo anterior suma un total de 134,253 USD. En relación al número de cabezas y el tipo de ganado depredado por especie, resalta que el oso negro y el 
puma depredan mayormente ganado caprino, el jaguar depreda mayormente ganado bovino, el coyote ganado caprino y el gato montés y la zorra gris predominan el ataque en ganado aviar (Tabla 1). Para el impacto económico sólo se integran en los resultados aquellos eventos de depredación que pudieron ser asociados a determinado depredador. Se tuvo cuidado de eliminar aquellos eventos que no coincidieran con patrones típicos de depredación, o que el encuestado no recordara detalles suficientes que permitieran la identificación del depredador.

\begin{tabular}{|c|c|c|c|c|c|c|c|c|c|c|}
\hline \multirow{2}{*}{ Especie } & \multicolumn{2}{|c|}{ Tipo de ganado } & \multirow[b]{2}{*}{ Mulas } & \multirow[b]{2}{*}{ Burros } & \multirow[b]{2}{*}{ Caprino } & \multirow[b]{2}{*}{ Aviar } & \multirow[b]{2}{*}{ Ovino } & \multirow[b]{2}{*}{ Porcino } & \multirow[b]{2}{*}{ Total } & \multirow{2}{*}{$\begin{array}{l}\text { Monto } \\
\text { \$ USD }\end{array}$} \\
\hline & Bovino & Caballos & & & & & & & & \\
\hline $\begin{array}{l}\text { Oso negro } \\
\text { (Ursus } \\
\text { americanus) }\end{array}$ & 128 & 3 & 0 & 10 & 276 & 0 & 126 & 6 & 549 & 43077.03 \\
\hline $\begin{array}{l}\text { Puma } \\
\text { (Puma concolor) }\end{array}$ & 57 & 11 & 1 & 14 & 101 & 0 & 0 & 0 & 184 & 17057.10 \\
\hline $\begin{array}{l}\text { Jaguar } \\
\text { (Panthera onca) }\end{array}$ & 179 & 9 & 0 & 6 & 49 & 0 & 4 & 0 & 247 & 39016.06 \\
\hline $\begin{array}{l}\text { Coyote } \\
\text { (Canis latrans) }\end{array}$ & 13 & 0 & 0 & 35 & 512 & 125 & 92 & 0 & 777 & 28492.86 \\
\hline $\begin{array}{l}\text { Gato montés } \\
\text { (Lynx rufus) }\end{array}$ & 0 & 0 & 0 & 0 & 55 & 69 & 34 & 0 & 158 & 4095.61 \\
\hline $\begin{array}{l}\text { Zorra gris } \\
\text { (Urocyon } \\
\text { cinereoargenteus) }\end{array}$ & 0 & 0 & 0 & 0 & 0 & 302 & 0 & 0 & 302 & 2514.80 \\
\hline
\end{tabular}

Daños por jaguar. Los resultados muestran que del total de entrevistados ( $n=80)$, sólo el $35 \%(n=28)$ reconocieron la presencia del jaguar en sus comunidades. Específicamente para jaguar, la suma obtenida de USD 39,016 corresponde a un total de 247 eventos registrados en el lapso de 1992-2010. La distribución de los daños por tipo de ganado atacado es: bovino-179 casos (registrados al menos 100 casos en edades de 5-8 meses); caprino-49 casos; caballar- nueve casos; burro- seis casos y ovino cuatro casos.

Discusión

Respecto a la característica de la muestra, el tamaño puede parecer bajo, pero si se toma en cuenta que es complicado que en esta zona las personas reconozcan el conflicto que existe con el jaguar, se considera un avance importante para el estudio con la especie, pues este número de encuestas se realizaron en un periodo de tres años de trabajo de campo, más dos de trabajo piloto. El acceso a información veraz lleva tiempo debido a que el equipo de trabajo tiene que ganar la confianza de las comunidades. Por otro lado, durante el desarrollo del trabajo de campo es común que una entrevista se realice en dos o tres sesiones en diferentes intervalos de tiempo, lo que lleva a profundizar más en la información y en el problema (Tarrés 2004). 
La persecución del jaguar por parte de los dueños del ganado, va de la mano con el nivel de daño que cada persona o familia haya sufrido (Soto-Shonder y Giulliano 2011; Garrote 2012). En ocasiones, el jaguar es perseguido ante el simple hecho de recibir información sobre su presencia en un sitio; eliminarlo se reconoce como medida preventiva en el problema de depredación. Cuando otro carnívoro que comparte el hábitat con el jaguar depreda ganado, es éste último quien frecuentemente es culpado por lo pobladores iniciándose su persecución (Garrote 2012). Conocer qué especie de carnívoro causa daños en el ganado, así como evaluar la magnitud de daño por una especie, es de suma importancia para iniciar trabajos de corte comunitario en los cuales se explique a los dueños del ganado cuestiones como la correcta identificación del depredador responsable de los daños (Hoogestijn y Hoogesteijn 2011).

En el área de estudio, los resultados de las encuestas muestran que el oso negro es el responsable de la cifra más alta de depredación, al igual que lo reportado por Pelton (1999) en toda su área de distribución en América, ubicándose como un depredador permanente para el ganado. El oso negro es una especie oportunista y consume desde venados, cultivos, basura o ganado si se da la oportunidad (Pelton 1999; Larivière 2001). Incluso, un elemento que surgió durante las encuestas fue la depredación de oso negro en los cultivos de maíz, durazno y manzana. Hay que resaltar no obstante, que existen vacíos de información con respecto a la situación de la población de esta especie que deben ser cubiertos (Delfín-Alonso et al. 2011) para poder asegurar que la depredación de ganado por oso negro se debe a un amento de la población y no por alteraciones de su hábitat natural. Dentro de este vacío de información es necesario indagar el efecto de los incendios, la alteración del hábitat natural para la implementación de cultivos y el aumento de la población humana en la dinámica poblacional de la especie como un detonante de la depredación de ganado.

Acerca de a la depredación de ganado por puma se tiene poca información publicada en México; un ejemplo de ello se da en Chihuahua donde el impacto de los pumas a la ganadería es bajo, siendo el ganado caprino uno de los más afectados (Bueno 2004), esto coincide con otros sitios en nuestro país como el centro y sureste de México (Monroy-Vilchis et al. 2009; Amador-Alcalá et al. 2013) donde el ganado caprino es preferido por el puma.

Respecto al gato montés y la zorra gris la depredación de ganado es un tema poco abordado. En la presente contribución se reporta depredación de aves de corral por ambos depredadores. Una de las pocas referencias es lo reportado por Amador et al. (2013) quienes encuentran que la zorra gris depreda exclusivamente aves de corral en el sureste mexicano, esto coincide con lo reportado de manera general en la literatura fuera de México para ambas especies (Nowell y Jackson 1996; Fritzell y Haroldson 1982; Fuller y Cypher 2004). Para la zona de la Ajusco en el Distrito Federal, Aranda et al. (2002), reportan que el gato montés depreda ganado ovino en bajas proporciones de su dieta.

El coyote se aborda de manera separada porque es considerado uno de los carnívoros más dañinos en la industria ganadera (Bekoff 1977; Gee 1979). En el presente trabajo el coyote aparece en tercer sitio en relación al impacto económico, en parte, porque muchas de las localidades donde se trabajó, están inmersas en las zonas montañosas en donde el coyote es menos abundante (Larivière y Walton 1997; Jiménez et al. 1999) a diferencia 
del Altiplano Mexicano o la Planicie Costera del Golfo en dónde estas cifras pudieran ser distintas. Los resultados de este trabajo coinciden con lo mencionado por Gee (1979) y Gese y Bekoff (2004) que reportan que el coyote depreda predominantemente ganado caprino y genera pérdidas millonarias en la industria ganadera (en 1974, se Ilegaron a reportar 16 millones de dólares en pérdidas) y es considerado una de las principales amenazas para los productores en la Unión Americana.

La depredación de ganado por jaguar en este estudio coincide con lo obtenido en otras contribuciones dónde el ganado representa una parte importante de la dieta del jaguar (Saenz y Carrillo 2002; Dalponte 2002; Garrote 2012). Los 247 casos obtenidos en este trabajo son superiores a los 31 casos obtenidos por Ortega (2006) en Querétaro, 70 casos de Hernández (2009) en Yucatán y un poco por debajo de los 270 casos que reportan Amador-Alcalá et al. (2013) en Chiapas. Estas diferencias muestran que la problemática del jaguar y el ganado es particular para cada una de las zonas de México y puede estar asociado a los sistemas de producción, a la identidad cultural de cada región e inclusive a las características biofísicas del lugar. Así también, en el tipo de ganado existen diferencias, para este trabajo el jaguar depreda predominantemente ganado bovino y en menor grado caprino, en comparación con Querétaro dónde reportan exclusivamente bovino. En Yucatán predomina la depredación sobre bovino y en menor grado ovino y en Chiapas la depredación de ganado caprino y ovino es dominante (Ortega 2006; Hernández 2009; Amador et al. 2013). La alta depredación de bovinos, puede ser una respuesta a que en el área predomina este tipo de ganado (INEGI 2007), y que pudiera ser de fácil acceso para el jaguar. Estos datos nos brindan información importante respecto a los programas de conservación del jaguar en México.

La estrategia nacional debe ser determinada de acuerdo a las características propias de cada región en el contexto socio cultural y biofísico.

Durante las conversaciones con los encuestados el jaguar fue mencionado como uno de los depredadores más dañinos (Peña-Mondragón 2011), aunque los resultados arrojan que el oso negro es responsable del número más alto de ataques en el ganado. Esto puede explicarse, quizás, por el impacto psicológico que causa en las personas la magnitud y violencia de los ataques de jaguar al ganado -predominantemente bovino-, debido a que se considera un elemento importante para la economía de las familias campesinas (funciona como un fondo de ahorro del que se puede disponer en determinadas emergencias, lo que les brinda un sentido de seguridad en el corto y mediano plazos; Chalate-Molina et al. 2010). En comparación, el ganado caprino y ovino comúnmente se utilizan para auto consumo o venta local, situación provocada por el bajo precio de venta de los hatos y por el papel desempeñado por los intermediarios (Hernández 2000). El alto costo de la depredación de jaguar en ganado bovino, provoca una persecución inmediata ante la presencia de la especie por parte de los pobladores, esto para evitar pérdidas futuras o detener y que no se extienda más el daño del jaguar en sus hatos ganaderos.

El problema de los daños causados en el ganado por jaguar, se puede analizar desde dos ópticas. Se puede examinar desde una visión limitada: el impacto por familia causado por el jaguar. Si se divide el costo de los daños totales causados por jaguar (39,016 USD) entre los 80 encuestados, se obtiene un valor de 487 USD por entrevistado. Esta cantidad dividida en un lapso de tiempo de 18 años, los daños de un jaguar para 
una familia por año resultan ser de 27 USD. Desde un segundo enfoque más integral que tome en cuenta no solamente al jaguar: los daños por familia no se limitan al daño por una sola especie. De acuerdo con los entrevistados, una familia puede sufrir daños a lo largo de un año por jaguar en su ganado bovino, pero también por coyote en su ganado caprino, y por oso en las siembras de maíz y/o el ganado ovino. Todo hay que sumarlo a los frecuentes y usualmente largos periodos de sequía o a inundaciones, desbarrancamiento de animales, enfermedades del ganado, así como al robo de ganado (factores no evaluados, pero que han surgido como relevantes en la zona de estudio según los resultados aquí expuestos). Todo esto permite visualizar el problema de forma diferente. Las pérdidas por daños de carnívoros, incluido el jaguar, suman cantidades que son relevantes para las familias campesinas.

Lamentablemente, desde la perspectiva campesina, la eliminación de carnívoros se percibe como una estrategia preventiva y necesaria, en las comunidades un individuo de cualquier especie de carnívoro es buscado y muchas veces eliminado con sólo detectar su presencia (Mishra 1997; Hoogesteijn 2003; Garrote 2012). Esto independiente de que haya causado daños o si ese individuo sea el responsable de los ataques de depredación si ya hubieran sucedido. En este sentido, el presente trabajo buscó abordar el conflicto de la depredación de ganado desde la perspectiva de las pérdidas económicas que sufren las comunidades campesinas en el caso del sur de Nuevo León, lo que permite entender que el conflicto va más allá de la simple depredación de ganado y que se trata de un problema multifactorial (Inskip y Zimmermann 2009). Los resultados aquí reportados, dan pautas para nuevas líneas de investigación como documentar la disponibilidad de presas naturales del jaguar, su dieta en la zona, evaluar el efecto de la cacería de subsistencia en las poblaciones de sus presas, evaluar pérdidas de ganado por otros factores que no sea depredación; pero también abre preguntas que se relacionan con conocer las percepciones y actitudes hacia la especie. Cabe resaltar, finalmente, que la información obtenida permite conocer y diferenciar el impacto de las diversas especies de carnívoros hacia los distintos tipos de ganado y esta información puede ser estratégica para las propias comunidades.

Reconocemos que el trabajo está dirigido a productores que han sufrido daños en su ganado por jaguar y otros carnívoros, pero eso responde a que pueden ser estos mismos productores los que toman represalias fatales contra las especies (Garrote 2012).

Queda mucho por hacer y es esencial diseñar estrategias de comunicación que apoyen en la formulación de intervenciones y políticas públicas de mitigación específicas para la diversidad de situaciones y lograr contribuir al mantenimiento a largo plazo de las poblaciones de carnívoros silvestres y en particular a la conservación del jaguar.

\section{Agradecimientos}

El primer autor agradece al Programa de Posgrado en Ciencias Biológicas de la Universidad Nacional Autónoma de México el apoyo brindado. Así mismo al Consejo Nacional de Ciencia y Tecnología (CONACYT) por la beca otorgada con número 333128/234322.

Agradecemos el apoyo económico proporcionado por The Rufford Foundation, sin el cual el trabajo no hubiera podido desarrollarse. Extendemos nuestro agradecimiento a los revisores anónimos que aportaron observaciones y críticas que han enriquecido el presente trabajo. Asimismo, agradecemos el apoyo técnico de H. Ferreira, A. López y 
A. Valencia, así como a E. de la Peña, R. Lombera, L. Martínez, H. Rodríguez-Vela, M. Zúñiga, J. Benítez, L. Reyna, J. Grimaldo, E. Cerda, G. Serrato y J. Medellín. Finalmente resaltar que agradecemos enormemente a cada uno de los pobladores que nos abrieron sus hogares y nos permitieron charlar y nos brindaron la información aquí presentada.

Este trabajo está dedicado a todos ellos.

Alanís, G., G. Cano, y M. Rovalo. 1996. Vegetación y flora de Nuevo León. Una guía Literatura citada botánico-ecológica, primera edición Impresora Monterrey. Monterrey, México.

Amador-Alcalá, S., E. J. Naranjo, y G. Jiménez-Ferret. 2013. Wildlife predation on livestock and poultry: implications for predator conservation in the rainforest of south-east Mexico. Oryx 47:243-250.

Aranda, M., O. Rosas, J. J. Ríos, y N. García. 2002. Análisis comparativo de la alimentación del gato montés (Lynx rufus) en dos diferentes ambientes de México. Acta Zoológica Mexicana 87:99-109.

BeкоғF, M. 1977. Canis latrans. Mammalian species 79:1-9.

Berkes, F., y C. Folke. 2000. Linking social and ecological systems. Management practices and social mechanisms for building resilience. Cambridge University Press. Cambridge, EE.UU.

Bueno, A. 2004. Impacto del puma (Puma concolor) en ranchos ganaderos del área natural protegida "Cañón de Santa Elena", Chihuahua. Tesis de Maestría. Instituto de Ecología, A. C. Xalapa, México.

Ceballos, G., C. Chávez, S. Blanco, R. Jiménez, M. López, O. Moctezuma, V. Támez y M. VALDEZ. 2006. Áreas prioritarias para la conservación. Pp. 13-19 in El jaguar Mexicano en el siglo XXI: Situación actual y manejo (Chávez, C., y G. Ceballos, eds). CONABIO-Alianza WWF Telcel-Universidad Nacional Autónoma de México. Ciudad de México, México.

Chalate-Molina, H., F. Gallardo-lópez, P. Pérez-Hernández, F. P. Lang-Ovalle, E. OrtegaJiménez, y J. Vilaboa-Arroniz. 2010. Características del sistema de producción bovinos de doble propósito en el estado de Morelos, México. Zootecnia Tropical 28:329-339.

Conde, D. A., F. Colchero, E. Huerta, C. Manterola, E.Pallares, A. Rivera, y A. Soler. 2011. El jaguar como elemento estratégico para la conservación. Comisión Nacional para el Conocimiento y Uso de la Biodiversidad. Ciudad de México, México.

Cortés, M. 2009. Diversidad de mamíferos medianos y grandes en dos sitios con diferente grado de conservación en La Venta, Juchitán, Oaxaca. Tesis de maestría. Instituto Politécnico Nacional. Oaxaca, Oaxaca.

Crawshaw, JR, P. G., y H. B. Quigley. 2002. Hábitos alimentarios del jaguar y el puma en el Pantanal, Brasil, con implicaciones para su manejo y conservación. Pp. 223-235 in El jaguar en el nuevo milenio (Medellín, R., C. Equihua, C. L. B. Chetkiewicz, P. G. Crawshaw Jr, A. R. Rabinowitz, K. H. Redford, J. G. Sanderson, y A. B. Taber, eds.). Fondo de Cultura Económica/Universidad Nacional Autónoma de México/ Wildlife Conservation Society. Ciudad de México, México.

DalPonte, J. C. 2002. Dieta del jaguar y depredación de ganado en el norte del Pantanal, Brasil. Pp. 209-235 in El jaguar en el nuevo milenio (Medellín, R., C. Equihua, 
C. L. B. Chetkiewicz, P. G. Crawshaw Jr, A. R. Rabinowitz, K. H. Redford, J. G. Sanderson, y A. B. Taber, eds.). Fondo de Cultura Económica/Universidad Nacional Autónoma de México/Wildlife Conservation Society. Ciudad de México, México.

De Azevedo, F. C. C. 2008. Food habits and livestock depredation of sympatric jaguars and pumas in the Iguacu National Park area, south Brazil. Biotropica 40:494-500

Delfín-Alfonso, C. A., C. A. López-González, y N. E. Lara-Díaz. 2011. El oso negro americano en el noroeste de México: recuperación de registros de ocurrencia. Acta Zoológica Mexicana 27:777-801.

Drury. R. , K. Omewood y, S. Randall. 2011. Less is more: the potential of quantitative approaches in conservation research. Animal conservation 14:18-24.

Fritzell, E. K., Y K. J. Haroldson. 1982. Urocyon cinereoargenteus. Mammalian species 189:1-8.

Fuller, T. K., y B. L. Cypher. 2004. Gray fox (Urocyon cinereoargenteus). Pp. 92-97 in Canids: foxes, wolves, jackals, and dogs. Status Survey and Conservation Action Plan (Sillero-Zubiri, C., M. Hoffmann, y D. Macdonald, eds.). IUCN/SSC Canids Specialist Group. Gland, Switzerland and Cambridg, UK.

Garrote, G. 2012. Depredación del jaguar (Panthera onca) sobre el ganado en los Ilanos orientales de Colombia. Mastozoología neotropical 19:139-145.

Gef, C. K. 1979. Cattle and calf losses to predators: feeder cattle enterprises in the United States. Journal of Range Management 32:152-154.

Gese, E. M., y M. Ekoff. 2004. Coyote (Canis latrans). Pp. 81-87 in Canids: foxes, wolves, jackals, and dogs. Status Survey and Conservation Action Plan (SilleroZubiri, C., M. Hoffmann, y D. Macdonald, eds.). IUCN/SSC Canids Specialist Group, Gland, Switzerland and Cambridg, UK.

Hernández-Sampierı, R. 1991. Metodología de la investigación. McGraw-Hill. Ciudad de México, México.

Hernández, Z. J. S. 2000. La caprinocultura en el marco de la ganadería poblana (México): contribución de la especie caprina y sistemas de producción. Archivos de zootecnia 49:341-352.

Hernández, A. D. 2009. Conflictos entre animales y humanos: la percepción de la depredación de ganado en Yucatán, México. Tesis de maestría. Centro de Investigación y de Estudios Avanzados del Instituto Politécnico Nacional.

Hoogesteijn, R. 2003. Manual sobre problemas de depredación causados por jaguares y pumas en hatos ganaderos. Wildlife Conservation Society, New York, EE.UU.

Hoogesteijn, R., y A. Hoogesteijn. 2011. Estrategias anti-depredación para fincas ganaderas en Latinoamérica: Una guía. PANTHERA. Gráfica y Editora Microart Ltda., Campo Grande, MS, Brasil.

InSkIP, C., y A. ZimmermanN. 2009. Human felid conflict: a review of patterns and priorities world. Oryx 43: 18-34.

Instituto Nacional de Estadística, Geografía, e Informática (INEGI). Censo Agropecuario. 2007. Disponible en línea: www.inegi.gob.mx (octubre 2013).

Instituto Nacional de Estadística, Geografía, e Informática (INEGI). Sintesís Geográfica. 1986. Nuevo León: Instituto Nacional de Estadística, Geografía e Informática. México. 
Jiménez, G. A., M. A. Zúñiga, y J. A. Niño. 1999. Mamíferos de Nuevo León, México. Universidad Autónoma de Nuevo León. Monterrey, México.

Larivière, S. 2001. Ursus americanus. Mammalian species 647:1-11.

Larivière, S., Y L. R. Walton. 1997. Lynx rufus. Mammalian species 563:1-8.

LONG , N. 1992. From paradigm lost to paradigm regained? The case for an actororiented sociology of development. Pp. 16-44. in Battlefields oh knowledge. The interlocking of theory and practice in social

López, R. H. 1998. La metodología de la encuesta. Pp. 33-73. in Técnicas de investigación en sociedad, cultura y comunicación (Galindo, C. J. J. ed.). Addison Wesley Longman, Ciudad de México, México.

meA (Millenium Ecosystem Assessment). 2003. Ecosystems and human well-being. A framework for assessment. Island Press. Washington, EE.UU.

Mishra, C. 1997. Livestock depredation by large carnivores in the Indian trans-Himalaya: conflict perceptions and conservation prospects. Enviromental Conservation 24: 338-343.

Monroy-Vilchis, O., Y. Gómez, M. Janczur, y V. Urios. 2009. Food niche of Puma concolor in central México. Wildlife Biology 15:97-105.

Moreno, R. S., y M. Oımos. 2008. Estudio preliminar sobre el problema de la depredación de ganado por jaguares (Panthera onca) y pumas (Puma concolor) en el Parque Nacional Portobelo, provincia de Colón, Panamá. Tecnociencia 10:85-98

Nowell, K., y P. Jackson (eds). 1996. Wild cats. Status Survey and Conservation Action Plan. IUCN, Gland, Switzerland and Cambridg, Reino Unido.

NOM-059-SEMARNAT-2001. Protección ambiental-Especies nativas de México de flora y fauna silvestres-Categorías de riesgo y especificaciones para su inclusión, exclusión o cambio-Lista de especies en riesgo.

Núñez, R., B . Miller, y F. Lindzey. 2002. Ecología del jaguar en la reserva de la Biosfera Chamela-Cuixmala, Jalisco, México. Pp. 107-126 in El jaguar en el nuevo milenio (Medellín, R., C. Equihua, C. L. B. Chetkiewicz, P. G. Crawshaw Jr, A. R. Rabinowitz, K. H. Redford, J. G. Sanderson, y A. B. Taber, eds.). Fondo de Cultura Económica/Universidad Nacional Autónoma de México/Wildlife Conservation Society. Ciudad de México, México.

OuiveirA, T. D. 1994. Neotropical cats: ecology and conservation. EDUFMA. São Luís, Brazil.

Ortega, A. M. 2006. Distribución y uso de hábitat del jaguar (Panthera onca) y el puma (Puma concolor) en la Rserva de la Biosfera "Sierra Gorda", Querétaro, México. Tesis de Maestría. Universidad Autónoma de Querétaro. Querétaro, México.

Palmeira, F. B., P. G. Crawshaw, C. M. Haddad, K. M. P. M. B. Ferraz, y L. M. Verdade. 2008. Cattle depredation by puma (Puma concolor) and jaguar (Panthera onca) in central-western Brazil. Biological Conservation 141:118-125

Pelton, M. R., A. B. Coley, T. H. Eason, D. L. Doan Martinez, J. A. Pederson, F. T. Van Manen, K. M. Weaver, y C. Servheen. 1999. American black bear conservation action plan. Pp. 144-156 in Bears. Status Survey and Conservation Action Plan (Servheen, C., S. Herrero, and P. Bernard, eds.). IUCN/SSC Bears Specialist Group. IUSN/SSC Polar Bear Specialist Group, Gland, Switzerland and Cambridg, UK. 
Peña-Mondragón, J. L. 2011. Daños económicos al ganado y percepciones sociales sobre el jaguar (Panthera onca veraecrucis Nelson and Goldman, 1933) en la Gran Sierra Plegada, Nuevo León, México. Tesis de maestría. Universidad Nacional Autónoma de México. Morelia, México.

Rosas-Rosas, O., y J. H. López-Soto. 2002. Distribución y estado de conservación del jaguar en Nuevo León, México. Pp. 393-401 in El jaguar en el nuevo milenio (Medellín, R., C. Equihua, C. L. B. Chetkiewicz, P. G. Crawshaw Jr, A. R. Rabinowitz, K. H. Redford, J. G. Sanderson, y A. B. Taber, eds.). Fondo de Cultura Económica/ Universidad Nacional Autónoma de México/Wildlife Conservation Society. Ciudad de México, México.

Saenz, J. C., E. Carrillo, y R. Medellín. 2002. Jaguares depredadores de ganado en Costa Rica: ¿un problema sin solución? Pp. 127-138 in El jaguar en el nuevo milenio (Medellín, R., C. Equihua, C. L. B. Chetkiewicz, P. G. Crawshaw Jr, A. R. Rabinowitz, K. H. Redford, J. G. Sanderson, y A. B. Taber, eds.). Fondo de Cultura Económica/Universidad Nacional Autónoma de México/Wildlife Conservation Society. Ciudad de México, México.

SánChez-Serrano, R. 2004. La observación participante como escenario y configuración de la diversidad de significados. Pp. 97-131 in Observar, escuchar y comprender. Sobre la tradición cualitativa en investigación social (Tarrés, M. L. ed.). Miguel Ángel Porrúa, FLACSO, El Colegio de México, Ciudad de México, México.

SAS Institute INC. 2007. JMP Software. Cary, NC: SAS Institute Inc. North Carolina, EE.UU.

Scognamillo, D., I. Maxit, M. Sunquist, y L. Farrell. 2002. Ecología del jaguar y el problema de la depredación de ganado en un hato de Los Llanos Venezolanos. Pp. 139-151 in El jaguar en el nuevo milenio (Medellín, R., C. Equihua, C. L. B. Chetkiewicz, P. G. Crawshaw Jr, A. R. Rabinowitz, K. H. Redford, J. G. Sanderson, y A. B. Taber, eds.). Fondo de Cultura Económica/Universidad Nacional Autónoma de México/Wildlife Conservation Society. Ciudad de México, México.

Secretaria de Medio Ambiente y Recursos Naturales (SEMARNAT). 2009. Programa para la conservación de la especie: Jaguar (Panthera onca). Ciudad de México, México.

Servín, J. y C. Huxuey. 1991. La dieta del coyote en un bosque de encino-pino de la Sierra Madre Occcidental de Durango, México. Acta Zoológica Mexicana 44:126.

Soto-Shoender, J. R., y W. M. Giuliano. 2011. Predation on livestock by large carnivores in the tropical lowlands of Guatemala. Oryx 45:561-568.

Tamang, B., y N. Baral. 2008. Livestock depredation by large cats in Bardia National Park, Nepal: Implications for improving park-people relations. The International Journal of Biodiversity Science and Management 4:44-53.

TARrés, M. L. (ed). 2004. Lo cualitativo como tradición. Pp. 35-60 en Observar, escuchar y comprender. Sobre la tradición cualitativa en investigación social (Tarrés, M. L. ed.). Miguel Angel Porrúa, FLACSO, El Colegio de México, Ciudad de México, México.

Vitousek, P., H. A. Mooney, J. Lubchenco, y J. M. Melillo. 1997. Human domination of Earth's ecosystems. Science 277:494-499 
WADE, D. A., Y J. E. Bowns. 1982. Procedures for evaluating predation on livestock and wildlife. Texas Agricultural Extension Service, Texas Agricultural Experiment Station, Texas A \& M University System, Texas. College Extension, EE.UU.

Sometido: 2 de septiembre de 2013

Revisado: 27 de octubre de 2013

Aceptado: 6 de novimbre de 2013

Editor asociado: Jorge Servin

Diseño gráfico editorial: Gerardo Hernández 
